\title{
The calibration of the RGB Tip as a Standard Candle
}

\section{Extension to Near Infrared colors and higher metallicity ${ }^{\star}$}

\author{
M. Bellazzini ${ }^{1}$, F. R. Ferraro ${ }^{2}$, A. Sollima ${ }^{1,2}$, E. Pancino ${ }^{1}$, and L. Origlia ${ }^{1}$ \\ 1 INAF - Osservatorio Astronomico di Bologna, via Ranzani 1, 40127 Bologna, Italy \\ e-mail: michele.bellazzini@bo.astro.it \\ 2 Dipartimento di Astronomia, Università di Bologna, via Ranzani 1, 40127 Bologna, Italy \\ e-mail: francesco.ferraro3@unibo.it
}

Received 19 December 2003 / Accepted 28 April 2004

\begin{abstract}
New empirical calibrations of the Red Giant Branch Tip in the $I, J, H$ and $K$ bands based on two fundamental pillars, namely $\omega$ Centauri and 47 Tucanae, have been obtained by using a large optical and near infrared photometric database. Our best estimates give $M_{I}^{\mathrm{TRGB}}=-4.05 \pm 0.12, M_{J}^{\mathrm{TRGB}}=-5.20 \pm 0.16, M_{H}^{\mathrm{TRGB}}=-5.94 \pm 0.18$ and $M_{K}^{\mathrm{TRGB}}=-6.04 \pm 0.16$ at $[M / H] \simeq-1.5(\omega \mathrm{Cen})$ and $M_{I}^{\mathrm{TRGB}}=-3.91 \pm 0.13, M_{J}^{\mathrm{TRGB}}=-5.47 \pm 0.25, M_{H}^{\mathrm{TRGB}}=-6.35 \pm 0.30$ and $M_{K}^{\mathrm{TRGB}}=-6.56 \pm 0.20$ at $[M / H] \simeq-0.6$ (47 Tuc).

With these new empirical calibrations we also provide robust relations of the TRGB magnitude in the $I, J, H$, and $K$ bands as a function of the global metallicity. It has also been shown that our calibrations self-consistently provide a distance modulus of the Large Magellanic Cloud in good agreement with the standard value $\left.\left((m-M)_{0} \simeq 18.50\right)\right)$.
\end{abstract}

Key words. distance scale - globular clusters: individual: NGC 5139; NGC 104 - stars: Population II - Magellanic Clouds

\section{Introduction}

The use of Tip of the Red Giant Branch (TRGB) as a standard candle is now a mature and widely used technique to estimate the distance to galaxies of any morphological type (see Lee et al. 1993; Madore \& Freedman 1995, 1998; Walker 2003, for a detailed description of the method, recent reviews and applications). The underlying physics is well understood (Madore \& Freedman 1998; Salaris et al. 2002) and the observational procedure is operationally well defined (Madore \& Freedman 1995). The key observable is the sharp cut-off occurring at the bright end of the RGB Luminosity Function that can be easily detected with the application of an edge-detector filter (Sobel filter, Madore \& Freedman 1995; Sakai et al. 1996) or by fitting the LF with a proper modeling function (Mendez et al. 2002). The necessary condition for a safe application of the technique is that the observed RGB Luminosity Function should be well populated, with more than $\sim 100$ stars within 1 mag from the TRGB (Madore \& Freedman 1995; Bellazzini et al. 2002).

The RGB develops to its full extent in stellar populations having age $\gtrsim 1-2$ Gyr, therefore the natural local systems with which the method may be calibrated are Galactic globular clusters (Da Costa \& Armandroff 1990; Ferraro et al. 2000). However, any calibration based on globulars must rely on the

\footnotetext{
* Based on observations collected at ESO, La Silla, Chile.
}

RR Lyrae (or Horizontal Branch) distance scale, whose zeropoint is still quite uncertain (Cacciari 1999; Walker 2003). Moreover the estimate of the TRGB in globular clusters is plagued by the low number of RGB stars available in each cluster, implying a considerable statistical uncertainty and forcing the adoption of a different operational definition of the observable (e.g., the brightest non-variable RGB star instead of the LF cut-off, see Da Costa \& Armandroff 1990; Ferraro et al. 2000; Bellazzini et al. 2001, and reference therein, for details and discussion).

To overcome these drawbacks Bellazzini et al. (2001, hereafter Paper I) used a large photometric database of the globular cluster $\omega$ Centauri (the most luminous in the whole Galaxy, therefore the one that has the largest number of RGB stars) and the distance estimate to this cluster by Thompson et al. (2001, based on the double-lined detached eclipsing binary OGLE 17), to provide an accurate zero point for the TRGB calibration in the Cousins $I$ passband, independent of the RR Lyrae distance scale. The detection of the cut-off in the LF of the upper RGB was clean and supported by a large sample comprising more that 180 stars in the brightest 1 mag of the RGB, e.g. fully satisfying all the requirements for a safe estimate of the TRGB magnitude as defined by Madore \& Freedman (1995) and Bellazzini et al. (2002) by means of numerical experiments. Furthermore the use of an essentially 
geometrical distance estimate to the considered cluster to calibrate the TRGB makes it, in practice, a primary standard candle. The calibration has already been applied by several authors in different contexts (Tosi et al. 2001; Maíz-Apellániz et al. 2002; Bellazzini et al. 2002; Alves 2003, 2004).

In the present paper we briefly re-discuss the analysis of Paper I in the light of some new results that have appeared in the literature and we extend the calibration to the Near Infrared (NIR) passbands with a statistically robust estimate of the absolute magnitude of the TRGB of $\omega$ Cen in the $J, H$ and $K$ bands based on the large NIR photometric database assembled by Sollima et al. (2003), complemented with 2MASS (Cutri et al. 2003) data. While the metallicity dependence of the magnitude of the TRGB is minimized in the Cousins $I$ band, the most suitable for distance estimates, the calibration of the standard candle in the NIR may prove very useful since (a) it may allow its safe application also in the cases of very high interstellar extinction, and (b) it will provide a natural tool for future studies performed with powerful telescopes optimized for infrared observations, as, for example, the James Webb Telescope that will push the application limit of the TRGB technique to much larger distances. Nevertheless, it has to be remembered that a safe application of the NIR calibrations requires a good estimate of the metallicity of the considered stellar system, which the TRGB brightness in these passbands ( $K$ and $H$ in particular) depends on very strongly.

To check our calibration also at different metallicity regimes (with respect to $\omega \mathrm{Cen}$ ) we obtained $B, V, I$ photometry of more that 100000 stars in a $\sim 0.5 \times 0.5 \mathrm{deg}^{2}$ field centered on the metal-rich globular cluster 47 Tucanae. With this new large sample, complemented with NIR photometry from Montegriffo et al. (1995) and 2MASS, we estimate the TRGB magnitude in the $I, J, H$, and $K$ bands also in this cluster.

The plan of the paper is the following: in Sect. 2 we briefly re-discuss the calibration of Paper I and we consider the possibility of extending the calibration to other clusters (of different metallicity with respect to $\omega$ Centauri); in Sects. 3 and 4 we present our new estimates of the absolute magnitude of the TRGB in the various passbands for $\omega$ Cen and 47 Tuc, respectively; in Sect. 5 we present the new calibrations of the TRGB in the $I, J, H$, and $K$ bands as a function of the global metallicity ([M/H], see Salaris et al. 1993) and we compare our derived distance scale with the "standard" distance modulus of the Large Magellanic Cloud $\left((m-M)_{0}=18.50\right.$, see Alves 2004). Finally, the main results of the paper are summarized in Sect. 6 .

\section{Refinements and extensions of the TRGB calibration}

The estimate of the apparent $I$ magnitude of the TRGB of $\omega$ Cen derived in Paper I is based on a very large sample (more than 220000 stars, see Pancino et al. 2000), the final observational uncertainties have been accurately estimated and can be considered to be as small as possible given the present technological resources.

On the other hand it was suggested in Paper I that the uncertainties in the other two ingredients of the obtained zero-point of the calibration, e.g. distance and reddening, may be significantly reduced with better data. For instance, according to Thompson et al. (2001), a better coverage of the velocity curve of OGLE-17 or a better estimate of the temperature of the members of the binary should significantly enhance the accuracy of the distance estimate. While no new results have become available since the publication of Paper I, there is interesting new information concerning the reddening. In a recent review Lub (2002) pointed out that while the $E(B-V)$ values usually assumed range from 0.09 to $0.16 \mathrm{mag}$, the actual reddening of $\omega$ Cen has been very accurately determined by a number of authors in the 70s and 80s. All the best independent methods consistently give $E(B-V)=0.11 \pm 0.01$, a more accurate and robust estimate than that adopted by Thompson et al. (2001) and in Paper I (e.g., $E(B-V)=0.13 \pm 0.02$ ). From now on we will follow the conclusions of Lub (2002), adopting $E(B-V)=0.11 \pm 0.01$.

First of all we repeated the analysis of Thompson et al. (2001) with the newly assumed reddening, to check the effect on the true distance modulus of $\omega$ Cen. The new estimate of the linear distance to the cluster is $D=5500 \pm 300 \mathrm{pc}$ (instead of $5350 \pm 300$ ). However, since the extinction enters in two of the equations needed to obtain the apparent distance modulus in $V$ (Eqs. (2) and (4) in Thompson et al. 2001), the effect of the new assumption cancels out and the final $(m-M)_{V}$ is unchanged, $(m-M)_{V}=14.04 \pm 0.11$. The true distance modulus is $(m-M)_{0}=13.70 \pm 0.11$ (instead of $\left.(m-M)_{0}=13.64 \pm 0.11\right)$, but the effect cancels out again in the determination of the absolute $I$ magnitude of the TRGB $M_{I}^{\mathrm{TRGB}}=-4.05 \pm 0.12$, just one hundredth of a magnitude brighter than that found in Paper I.

The distance modulus derived above is in excellent agreement with the most recent estimates obtained with different methods, e.g. the HB semi-empirical fitting by F99 and Rey et al. (2000), the theoretical Period-Luminosity-Metallicity relation used by Caputo et al. (2002) and the RR Lyrae distance scale obtained by Gratton et al. (2003) from the more recent and finest application of the Main Sequence fitting of galactic globulars to local subdwarfs whose distance has been measured with the Hipparcos satellite (see also Olech et al. 2003). Hence the fundamental pillars of our calibration seem to have gained further support from the advancements made in this field since the publication of Paper I.

\subsection{Metallicity, age and contamination issues}

It is well known that $\omega$ Cen is not a Simple Stellar Population (SSP, Renzini \& Fusi Pecci 1988) but instead it host stars of different metallicities and ages (see Norris \& Da Costa 1995; Norris et al. 1996; Suntzeff \& Kraft 1996; Hilker \& Richtler 2000; Hughes \& Wallerstein 2000; Pancino et al. 2000; Pancino 2003 and references therein). In Paper I we briefly discussed the main reasons that led us to consider this stellar system as a suitable pillar of the TRGB calibration in spite of these possible shortcomings. Here we illustrate these arguments in greater detail, considering the potential problems associated with the presence of a metallicity and age spread and with foreground contamination by stars in the Galactic disc. 
Several photometric and spectroscopic studies have ascertained that $\omega$ Cen hosts stars of widely different chemical abundances, from $[\mathrm{Fe} / \mathrm{H}] \simeq-2.0$ to $[\mathrm{Fe} / \mathrm{H}] \simeq-0.5$ (Norris \& Da Costa 1995; Pancino et al. 2002; Origlia et al. 2003). However, the results of the largest spectroscopic surveys clearly indicate that the metallicity distribution of $\omega$ Cen is characterized by an obvious main peak around $[\mathrm{Fe} / \mathrm{H}] \simeq$ -1.7 (corresponding to $[\mathrm{M} / \mathrm{H}] \simeq-1.45$, see Sect. 5, below). From the spectroscopic metallicity estimates for 144 RGB stars by Suntzeff \& Kraft (1996) it can be concluded that more than $80 \%$ of the cluster stars are enclosed in the narrow range $[\mathrm{Fe} / \mathrm{H}]=-1.7 \pm 0.2$. The same conclusion can be drawn from the survey by Norris et al. (1996) of 517 RGB stars and from the large set of metallicities obtained from Strömgren photometry by Hilker \& Richtler (2000). Hence, it can be concluded that the stellar content of $\omega$ Cen is largely dominated by a stellar population spanning a narrow range in metallicity (and presumably age) and it can be safely considered as an approximate SSP in the present context. In Sect. 3.1, in the process of the detection of the TRGB in the NIR passbands, we will select RGB stars by their colors to avoid possible contamination by stars not associated with the main peak of the metallicity distribution.

The weak sensitivity of the TRGB luminosity to age for ages larger than $\sim 4$ Gyr constitutes the original theoretical basis of its use as a standard candle. At a given chemical composition the TRGB level is determined by the mass of the stellar core at the helium flash, which is fairly constant over a large part of the low-mass star range (see Salaris et al. 2002; Madore \& Freedman 1998, and references therein). Note that this is the same basic reason that allows the use of RR Lyrae and/or Horizontal Branch stars as standard candles. Using either the models adopted by Salaris \& Cassisi (1998) or the analytical formulae provided by Rood \& Crocker (1997) or by Buzzoni $(1989,2002)$, we easily recover the well known result that the bolometric magnitude $\left(M_{\mathrm{bol}}\right)$ of the TRGB decrease by less than $0.1 \mathrm{mag}$ while the age varies from $14 \mathrm{Gyr}$ to $4 \mathrm{Gyr}$ (our analysis is limited to stellar populations with less-thansolar metallicity). We also considered the impact of bolometric corrections (BC), adopting the prescriptions by Da Costa \& Armandroff (1990) for the Cousins I passband and those by Montegriffo et al. (1995) for $J$ and $K$. We found that the difference in bolometric correction at the TRGB due to age differences in the considered range (a) is less than 0.05 mag in $I$ and $J$ and less than 0.2 mag in $K$, and (b) in $J$ and $K$ (and presumably also in $H$ ) goes in the opposite direction with respect to the behavior of $M_{\mathrm{bol}}$. It turns out that, at any metallicity, the magnitude of the TRGB in the $J$ passband is the less sensitive to age variations and that, in any of the considered passbands, the TRGB magnitude varies less than $0.06 \mathrm{mag}$ for an age difference of $6 \mathrm{Gyr}$, and less than $0.12 \mathrm{mag}$ for an age difference as large as $10 \mathrm{Gyr}$.

Even more relevant, in the present context, is the recent analysis by Barker et al. (2004). These authors studied the dependence of the TRGB level of composite stellar populations on the adopted star formation history (SFH) by means of extensive experiments with synthetic CMDs. They conclude that "...the TRGB-distance method is insensitive to star formation history except for large bursts between ages of about 1 and 2 Gyr". The maximum age spread suggested by Hilker \& Richtler (2000) and Hughes \& Wallerstein (2000) for $\omega$ Cen is $\leq 6$ Gyr and the stars younger than the metal-poor bulk of the cluster stars are a small fraction of its whole population. Hence we should conclude that the observed age spread is not a concern for the use of $\omega$ Cen as a fundamental pillar of our TRGB calibration. The considerations above also demonstrate that age (or SFH) issues should not be a concern even for the application of the method to the Large Magellanic Cloud, presented in Sect. 5.1, below.

Finally, the relatively low Galactic latitude of $\omega$ Cen $\left(b \simeq 15^{\circ}\right.$ ) implies significant contamination of the CMD by foreground/background stars belonging to the Galactic disc. Nevertheless, the inspection of the available CMDs (Pancino et al. 2000) suggests that disc contamination is not a serious problem at the TRGB level for fields like the ones considered here. According to the Galactic model by Robin et al. (2003), the field stars expected in the upper 1 mag from the TRGB of $\omega$ Cen and with similar color as the cluster RGB are less than 20 and they are nearly uniformly distributed in magnitude. Hence they cannot have any sizeable influence on the clear detections of the cut-off of the RGB LF of $\omega$ Cen shown in Paper I and in Sect. 3.1, below.

\subsection{Searching for other pillars: The case of 47 Tuc}

The criterion for a safe detection of the TRGB based on $N_{\star}$ (Madore \& Freedman 1995; Bellazzini et al. 2002) may be fulfilled by collecting the photometry of large samples of RGB stars. The task may be achieved by observing sufficiently wide regions of the stellar system under consideration, for instance by using a wide field instrument, as done in Paper I. However, insuperable intrinsic obstacles to this aim may be encountered, e.g. the considered stellar system may not have a sufficient number of RGB stars. In a Simple Stellar Population (SSP, composed of stars of the same age and chemical composition, like a globular cluster - Renzini \& Fusi Pecci 1988) the number of stars in a given evolutionary phase at any given time scales with the total luminosity according to the Evolutionary Flux relation (Renzini \& Buzzoni 1986). For example, according to Renzini (1998), a 15 Gyr old SSP of solar metallicity having a total luminosity of $10^{5} L_{\odot}$ should have just 10 RGB stars within 1 mag from the TRGB, clearly insufficient for a reliable detection of the observable.

This basic consideration indicates that Galactic globulars in which a safe detection of the TRGB is possible are rare ${ }^{1}$.

In Paper I we found $N_{\star} \simeq 185$ for $\omega$ Cen, by far the most luminous globular cluster of the whole Milky Way. Scaling this number with the ratio of cluster luminosity (taken from

1 In particular if the adopted observable is the cut-off in the RGB LF. This observable is much more reliable than "the brightest non-variable star" when a distant stellar system is considered, since it does not need an exact discrimination between AGB and RGB stars (often not possible in such systems) and it is not sensitive to the contamination by background and foreground stars, if the degree of contamination is not extreme (Madore \& Freedman 1995). 
Harris 1996) to the luminosity of $\omega$ Cen we find only three more clusters for which a complete sampling of the RGB stars would achieve the condition $N_{\star} \gtrsim 100$, e.g. NGC 6715 , NGC 6441 and NGC 2419. Each of them presents intrinsic problems: the CMD of NGC 6715 is strongly contaminated by stars of the Sgr dwarf galaxy, in which it is embedded (Sarajedini \& Layden 1995); NGC 6441 is located in the Galactic bulge and suffers from strong (and uncertain) extinction $(E(B-V)=0.47$, Harris 1996); NGC 2419 is one of the most distant Galactic clusters known. For none of them is a RR Lyrae (or HB)-independent distance estimate presently available. These factors make these cluster unsuitable as safe pillars of the TRGB calibration, at least at the present stage.

Bellazzini et al. (2002) provided a relation to correct the estimate of $I_{\mathrm{TRGB}}$ for low-sample effects in the range $N_{\star} \gtrsim 80$, thus making it possible to include slightly less luminous clusters among the potential calibrators at the expense of a larger uncertainty in the final estimate. With this extension, the most natural candidate is certainly 47 Tucanae (NGC 104) that is relatively luminous, nearby, has modest extinction and is one of the most extensively studied clusters in the whole Galaxy. In Sect. 4 we will describe the detection of the TRGB in this cluster, based on a newly obtained photometric dataset including the large majority of the 47 Tuc giants.

\section{The TRGB of $\omega$ Cen in the $J, H$ and $K$ bands}

To obtain a NIR photometric database of comparable size with respect to that used in Paper I we merged the $J, K$ photometry by Sollima et al. (2003, hereafter S03) with $J, H, K$ photometry of a circular region centered on $\omega$ Centauri and with $1 \mathrm{deg}$ radius (of the order to the cluster tidal radius, see van Leeuwen et al. 2000; Harris 1996, and references therein) taken from the Point Source Catalogue of 2MASS. The photometry by S03 covers a field of $\simeq 13 \times 13 \operatorname{arcmin}^{2}$ in the central region of the cluster and provides much deeper and accurate photometry than the 2MASS catalogue. Selecting the sources from the latter we accepted only first quality stars, with no indication of blending, corruption or problems in the measurements. These requirements make the $2 \mathrm{MASS}$ catalogue very incomplete in the inner $10^{\prime}$ where crowding is too severe for the low spatial resolution of the survey camera. Therefore the two merged catalogues are nicely complementary, covering different radial annuli of the cluster from the center to the limiting radius. The tiny photometric shifts described by S03 have been applied to the 2MASS catalogue to match the photometric system of S03. The comparison between the stars in common between the two catalogues suggest that the final photometries are on the same photometric system within $\pm 0.03 \mathrm{mag}$ in $J$ and $K$ and that the star to star scatter at any magnitude is $\lesssim 0.1 \mathrm{mag}$. These figures have to be considered as upper limits since the comparison is necessarily performed in the small overlapping region in which crowding may still sensibly affect the accuracy of 2MASS photometry of individual stars. In the final merged catalogue we used the 2MASS dataset to complement the S03 catalogue both in radial extension (all the stars outside the S03 field) and in photometry ( $H$ band measurements not included in the S03 catalogue).

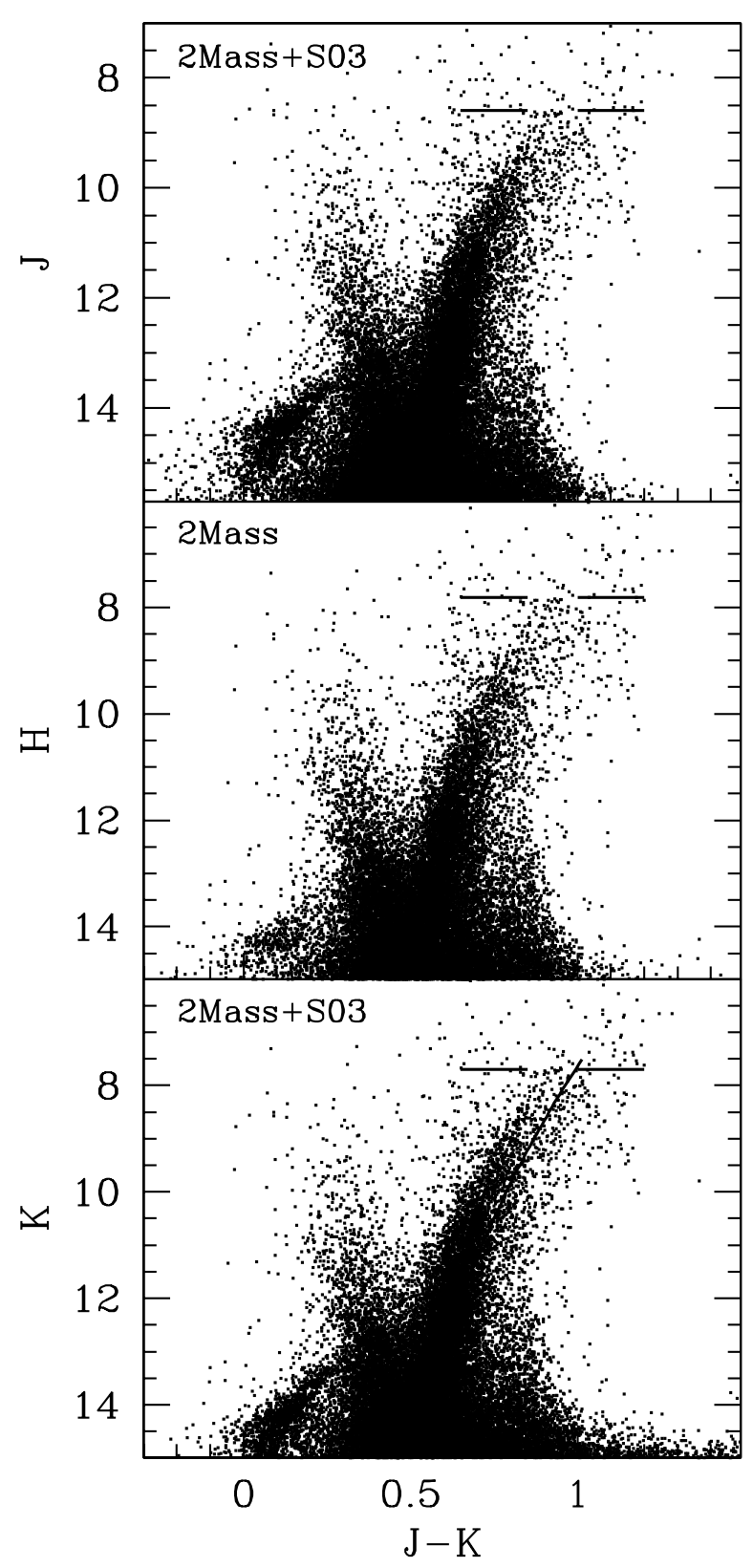

Fig. 1. NIR CMDs of $\omega$ Centauri. The position of the TRGB is marked by the two segments. In the lower panel we also plotted the RGB ridge-line of the cluster $\mathrm{M} 13([\mathrm{M} / \mathrm{H}]=-1.2 ;[\mathrm{Fe} / \mathrm{H}]=-1.4)$ taken from Valenti et al. (2004). For the TRGB detection we selected only RGB stars to the blue of the reported ridge-line, in order to avoid contamination by metal-rich stars.

\subsection{Detection of the TRGB}

In the color range $-0.3<J-K<1.3$ the final catalogue contains 72419 sources with $6.5<K<18$ taken from S03 and 47000 sources with $6.5<K \lesssim 16$, i.e. a total of $\sim 120000$ stars. The RGB stars brighter than $K=11$ are 2035, viz. 940 from S03 and 1456 from the 2MASS catalogue.

The resulting NIR Color Magnitude Diagrams (CMDs) are presented in Fig. 1. The RGB of $\omega$ Cen is the most evident feature of these diagrams at $0.5 \lesssim J-K \lesssim 1$ and ranging from $K \sim 14$ to $K \sim 7.5$. The Blue Horizontal branch of the 


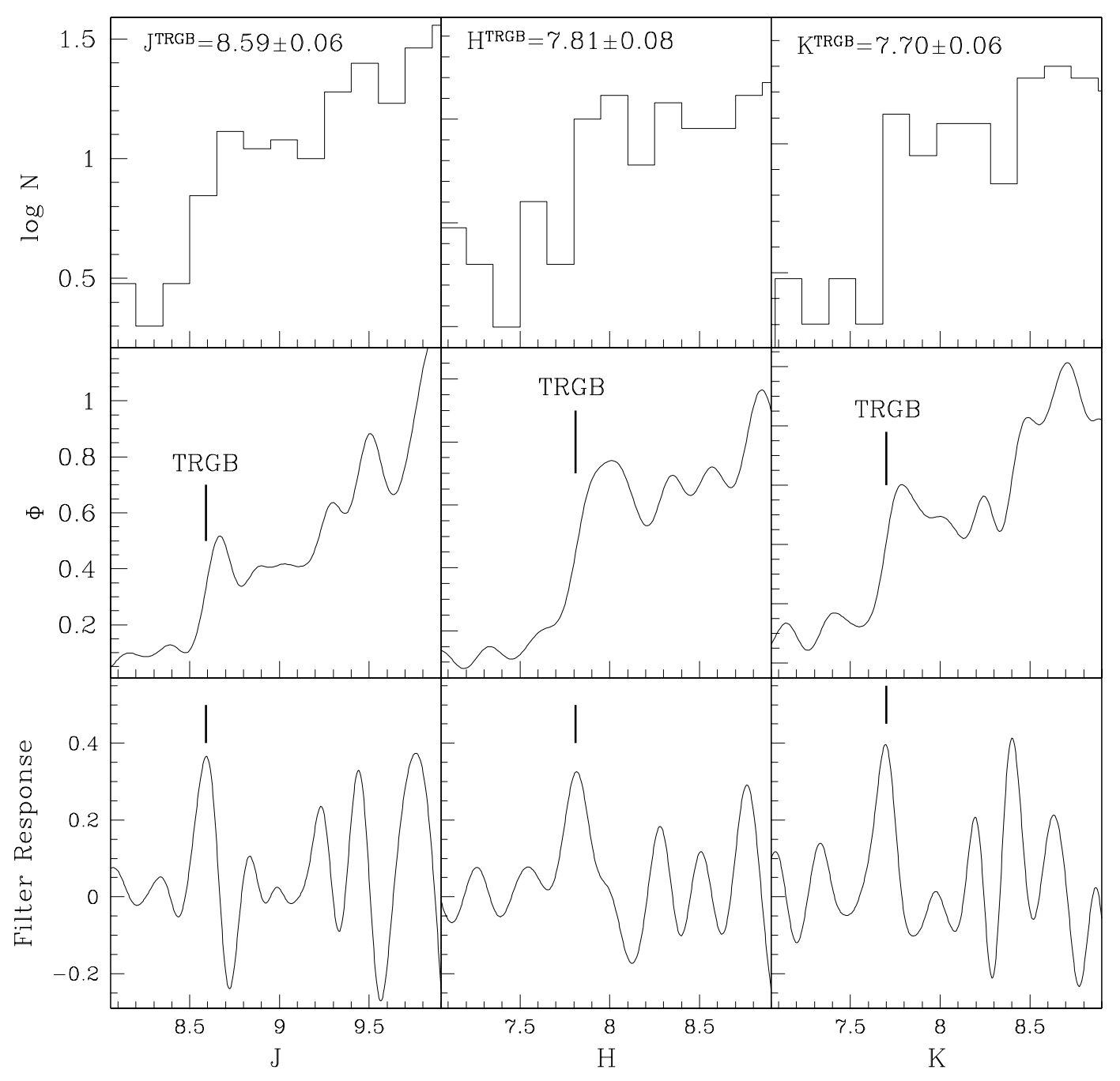

Fig. 2. Detection of the TRGB in the $J, H$, and $K$ bands from the upper RGB LF of $\omega$ Centauri. The upper panels report the LF as an ordinary log histogram, the middle panels show the generalized histogram (i.e. the histogram in the upper panels convolved with a Gaussian with standard deviation equal to the photometric error at the given magnitude, see Bellazzini et al. 2002, for definitions and references) and the lower panels display the response of the Sobel filter to the observed LF. The position of the TRGB is indicated by a thick vertical segment.

cluster is also visible at $K<13$ and $J-K \lesssim 0.4$. The Asymptotic Giant Branch does not appear clearly in these diagrams because of the unfavorable scale but it is also clearly identified in the database (see S03 for enlarged CMD). The blue plume around $J-K \sim 0.3$ and extending to $K \sim 8$ is due to foreground Main Sequence stars of the Galactic Disc. The level of the TRGB (as derived below) is also reported in all CMDs of Fig. 1. Approximately half of the red sources $(J-K \sim 1)$ brighter than the TRGB are expected to be foreground field sources, according to the predictions of the Galactic model by Robin et al. (2003). Some of them may be blended sources, since the large majority comes from the lowspatial-resolution 2MASS catalogue. The fact that our final estimates of the TRGB level in NIR passbands are unaffected if we limit the analysis to the high-spatial-resolution S03 sample ensures that the possible contamination by blended sources from the 2MASS catalogue is not a serious concern in the present application. An exhaustive analysis of these stars is clearly beyond the scope of the present study. The key point here is that the cut-off of the RGB population marking the location of the Tip is evident in the CMD of Fig. 1 as well as in the Luminosity Functions shown in Fig. 2 below, hence these "supra-TRGB" stars do not appear to affect the actual detection of the Tip. The main argument at the basis of the use of RGB-LF cut-off as the marker of the TRGB level is that it is essentially insensitive to the effects of contaminating populations of any kind, once a well sampled RGB is considered.

The detection of the TRGB in the three NIR passbands has been performed with the same technique used in Paper I (Lee et al. 1993; Madore \& Freedman 1995, 1998) and is described in Fig. 2. The upper panels of the plot show the log LF of the upper RGB as an ordinary histogram while middle panel shows the LF as a generalized histogram (see Paper I and references therein). The sharp drop in the LF is clearly visible in both histograms and it is neatly detected by the Sobel filter whose response is plotted in the lower panels of Fig. 2. The position of the TRGB is marked by a thick vertical segment. The apparent magnitude of the TRGB is $J=8.59 \pm 0.06, H=7.81 \pm 0.08$, 
and $K=7.70 \pm 0.06$, respectively. The reported uncertainties are the half width at half maximum of the peaks of the Sobel filter response.

Because of the larger sensitivity to metallicity of the TRGB in $J, H$ and $K$ with respect to $I$, we were forced to search the cut-off in the LF of the blue bulk of the RGB population, excluding the reddest stars that have metallicity $[\mathrm{Fe} / \mathrm{H}] \gtrsim-1.4$ (Pancino et al. 2000; Ferraro et al. 2002; Pancino 2003, and references therein). Such a selection (illustrated in the lower panel of Fig. 1) reduced the number of RGB stars in the upper part of the LF with respect to the analysis of Paper I. We made a number of simulations using subsamples of our RGB stars to estimate the TRGB in the same way shown in Fig. 2 and we find that the best estimate is recovered within \pm 0.04 mag in more than $95 \%$ of the cases if $N_{\star}>50$, independently of the considered NIR passband. For our best estimates (e.g. those presented in Fig. 2) $N_{*}=100,69,86$ in $J, H$, and $K$, respectively, hence it is very unlikely that they are significantly affected by small-sample problems. As stated, the results are also unchanged if we limit the analysis to the S03 sample.

Adopting the distance modulus and reddening described in the previous section, and assuming the reddening laws by Rieke \& Lebofsky (1985) we obtain $M_{J}^{\mathrm{TRGB}}=-5.20 \pm 0.16$, $M_{H}^{\mathrm{TRGB}}=-5.94 \pm 0.18$ and $M_{K}^{\mathrm{TRGB}}=-6.04 \pm 0.16$, where the error bars account for all the sources of uncertainty ${ }^{2}$.

\section{The TRGB of 47 Tuc in $I, J, H$ and $K$}

We retrieved from the ESO archive seven 60s images for each filter $(B, V, I)$ centered on the center of 47 Tucanae. The images were taken with the ESO-MPI-WFI camera mounted at the $2.2 \mathrm{~m}$ telescope at La Silla (Chile) as part of the Pre-Flames ESO Imaging Survey (EIS) ${ }^{3}$. Since the upper RGB stars of the cluster were saturated in the EIS images, we complemented the observational material with one proprietary $20 \mathrm{~s}$ exposure for each filter taken with the same instrument. The seeing of the images ranges from 0.8 arcsec to $1.5 \mathrm{arcsec}$ with a median of $\sim 1.1$ arcsec. The images were corrected for bias and flat-field with standard IRAF procedures and the photometry of each was obtained independently using DoPhot (Schechter et al. 1993). For each passband the catalogues from the seven $60 \mathrm{~s}$ exposures were cross-correlated, reported to the same (instrumental) photometric system and the stars in common were averaged with the additional requirement that a star is included in the final catalogue only if it is successfully measured in at least 3 images per filter. The averaged $B, V, I$ catalogues were finally combined and merged with the catalogue obtained from the short exposures, after transferring the latter to the same photometric system. All the stars in the total catalogue with $I \lesssim 12$ are from the $60 \mathrm{~s}$ exposures while those brighter than this limit are from the $20 \mathrm{~s}$ exposure. The long/short exposure threshold is located approximately at the base of the AGB clump. The absolute calibration was obtained by direct comparison with the large set

\footnotetext{
${ }^{2}$ In the following we will always adopt the reddening laws by Rieke \& Lebofsky (1985) for the $J, H$, and $K$ bands and Dean et al. (1978) for the Cousins $I$ passband.

${ }^{3}$ http://www.eso.org/science/eis/surveys/ strategy_Pre-Flames.html
}

of secondary standard stars provided by Stetson (2000) that are present in our field. More details about the dataset will be provided in a future paper devoted to a more thorough study of the cluster, while here we are interested only in the TRGB. The final optical catalogue contains 105745 stars in the range $0.0<V-I<3.0$ and $8.5 \leq I<21$.

The NIR dataset was assembled in a similar way to that for $\omega$ Cen described above. $J, K$ photometry for the inner $4 \times 4 \operatorname{arcmin}^{2}$ was taken from Montegriffo et al. (1995, hereafter $\mathrm{M} 95$ ) and $J, H, K$ photometry of a circle centered on the cluster of radius $r=33^{\prime}$ was retrieved from the PSC of 2MASS with the same requirements and transformations as those adopted for $\omega$ Cen. There are very few stars in the inner $\simeq 6^{\prime}$ in the 2 MASS catalogue, hence there is a nearly annular region between $r \sim 4^{\prime}$ and $r \sim 6^{\prime}$ that is not sampled by our total NIR catalogue. Therefore, in the case of 47 Tuc, the statistics of RGB stars in the NIR could be improved by observations that cover also this area. In our final NIR catalogue there are more than 4000 stars with $K<15$. There are 445 RGB stars with $K<11,240$ of which are from M 95 and 205 from the 2MASS catalogue. Finally we identified all the Long Period Variables (LPV) from the lists by M 95 and Clement (1997) that are present in our sample (namely V1, V2, V3, V4, V6, V7, V8, V11, V13, V19, V20, V21, V22, V26, V27, A19).

To illustrate the properties of our sample we plot in Fig. 3 some examples of optical, optical-infrared and infrared CMDs. The RGB is well defined in every plane and it is clearly separated from the red $\mathrm{HB}$ and from the AGB clump and sequence. The red plume that crosses the cluster MS in the $(I, V-I)$ diagram is the RGB of the Small Magellanic Cloud that lies in the background of our field.

\subsection{Detection of the TRGB}

Figure 4 illustrates the detection of the TRGB in the $I$ passband, obtained with the same technique as adopted in Paper I and in Fig. 2. We find $I_{\mathrm{TRGB}}=9.49 \pm 0.1$. Since $N_{\star}=80$ we have to correct our estimate with the relation provided by Bellazzini et al. (2002). In the present case the correction is $\delta I_{\mathrm{TRGB}}=$ $-0.09 \pm 0.08$, hence our final estimate is $I_{\mathrm{TRGB}}=9.40 \pm 0.13$. The corrected position of the TRGB is also reported in Fig. 4.

The NIR database for 47 Tuc is significantly smaller than the optical one and the number of RGB stars sampled is insufficient for a safe application of the technique adopted above. Hence we are forced to use the brightest non-variable RGB star as a tracer of the TRGB. However, in doing this, we take advantage of the robust detection we obtained in $I$ by imposing self-consistency among the estimates in the various passbands. In practice, we require that the stars marking the position of the TRGB shall be the same in any of the considered filters.

The procedure followed is illustrated in Fig. 5 in which the CMDs of the upper RGB are plotted in the various optical/IR planes. The are two non-variable stars which lie very close to the position of the TRGB. We use these two stars as tracers of the TRGB position in $K$ and $J$, taking their average magnitude as the best estimate for the TRGB and adding in quadrature the standard deviation to the uncertainty in $I_{\mathrm{TRGB}}$. 
47 Tucanae
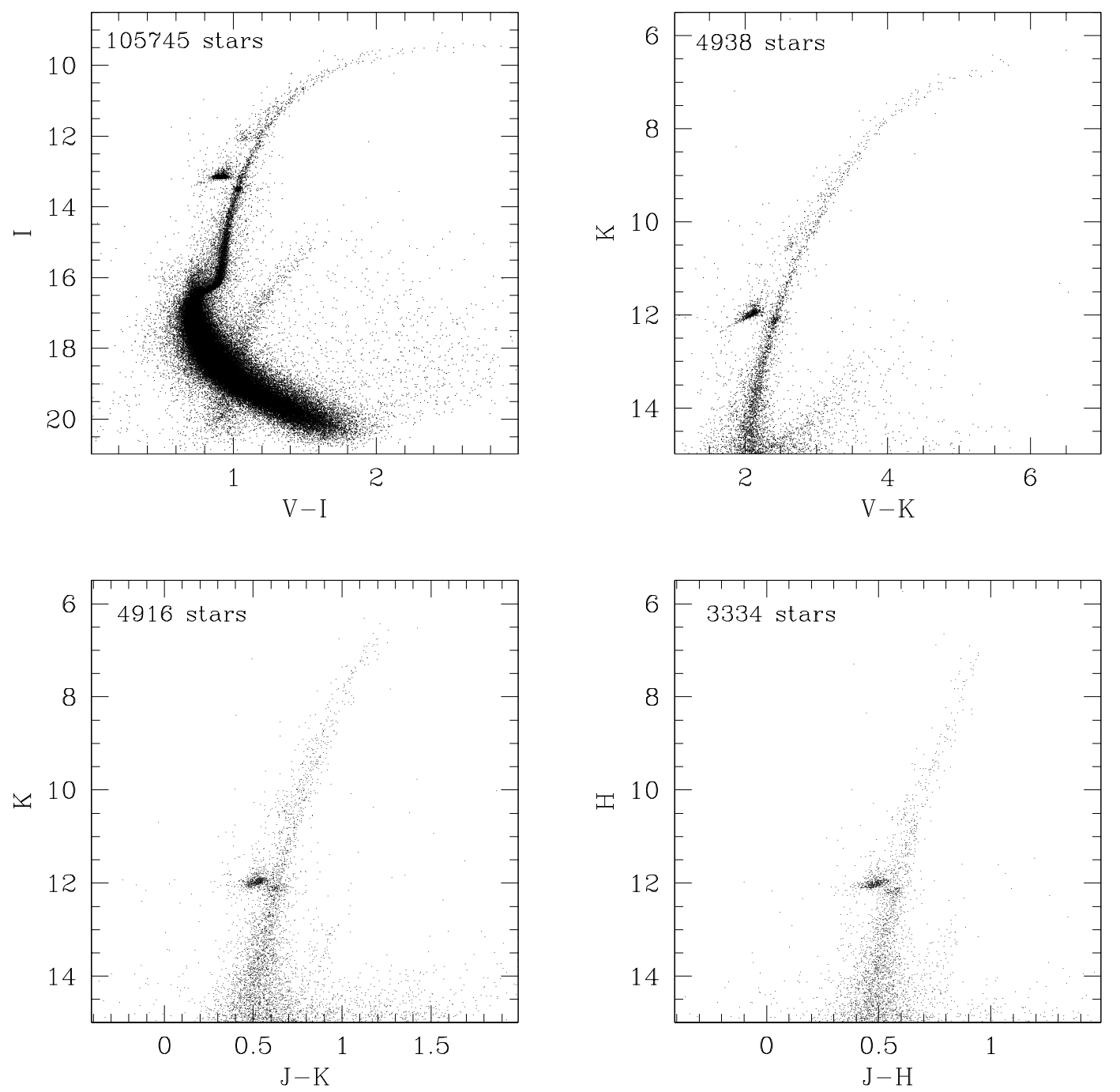

Fig. 3. Optical and NIR CMDs of 47 Tucanae obtained from our photometric sample implemented with the 2MASS database and the $J$, $K$ photometry by Montegriffo et al. (1995). The feature at $I>14$ and $K>12$, mostly to the red of the cluster CMD is the RGB of the Small Magellanic Cloud that lies in the background.

Note that in the $K$ passband the two stars have nearly the same magnitude. Note also that the selected stars are the brightest non-variable stars in any of the considered passbands. Hence our choice of the "TRGB markers" is fully justified independently of the operational definition actually adopted, e.g. the RGB-LF cut-off or the brightest non-variable star.

Unfortunately these stars lack $H$ photometry. The brightest non-variable star with $H$ photometry is marked by a cross in the CMDs of Fig. 5. It can be appreciated that this star is $0.1 \mathrm{mag}$ fainter than the TRGB level in $K$. It is reasonable to assume that a similar underestimate of the TRGB level should occur also in the $H$ band, therefore, as our best estimate of $H_{\mathrm{TRGB}}$ we take the $H$ magnitude of the considered star shifted by -0.1 mag.

It is obvious that the estimates of the observed TRGB level of 47 Tuc described above suffer from larger uncertainties than do those for the case of $\omega$ Cen. We take into account this fact by conservatively combining all the sources of uncertainty in the final calibrations, including the uncertainty associated with $I_{\mathrm{TRGB}}$ which drove our detections in the NIR bands. Our final estimates are: $J_{\mathrm{TRGB}}=7.84 \pm 0.25, H_{\mathrm{TRGB}}=6.96 \pm 0.30$, and $K_{\mathrm{TRGB}}=6.75 \pm 0.20$.

\subsection{Comparison with previous analysis}

It is interesting to consider the impact of the adoption of our large database on the estimate of the TRGB level from a purely observational point of view. The brightest non-variable star of 47 Tuc in the original DA90 sample has $I=9.65$, while in the final sample adopted by DA90 (implemented with photometry from other sources) the brightest non-variable star has $I=9.50$, that is 0.25 and 0.01 mag fainter, respectively, than our observed value $(I=9.49)$, before any correction for under-sampling $(\delta I)$. If the corrected estimate is considered $(I=9.40)$, it has to be concluded that the previous estimate 


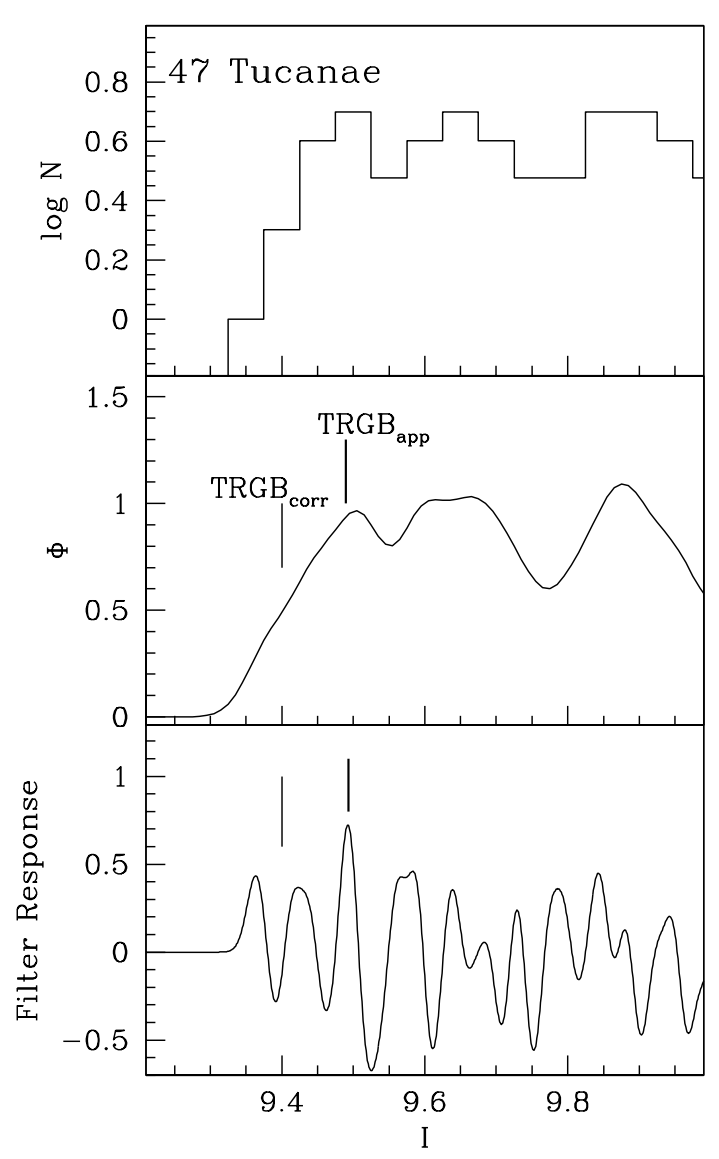

Fig. 4. Detection of the TRGB in $I$ for 47 Tucanae, with the same technique as displayed in Fig. 2. The apparent $\left(\right.$ TRGB $\left._{\text {app }}\right)$ and corrected $\left(\mathrm{TRGB}_{\text {corr }}\right)$ positions of the TRGB are also shown.

of $I_{\mathrm{TRGB}}$ for 47 Tuc missed the true TRGB level by $\sim 0.2 \mathrm{mag}$ because of the severe under-sampling of the upper RGB population (see Paper I and references therein). The effect is not so large for the NIR passband, since the dataset of Montegriffo et al. (1995) contains many more RGB stars than there are in the optical sample used by DA90, and the bulk of the evolved stars in our catalogue are taken from Montegriffo et al. (1995). Our estimate of $K_{\mathrm{TRGB}}$ coincides with that by Montegriffo et al. (1995), while our $J_{\text {TRGB }}$ is 0.1 mag brighter than their estimate. No previous estimate of $H_{\mathrm{TRGB}}$ is available in the literature. Hence, despite the considerable uncertainties that still affect the estimates of the TRGB level of 47 Tuc presented in this paper, they represent significant progress with respect to those previously available.

The above example shows in practice that the derivation of $I_{\mathrm{TRGB}}$ from small samples may provide a serious underestimation of the TRGB luminosity. Hence, it may appear surprising that the $M_{\mathrm{TRGB}}^{I}$ calibration by DA90 - whose original estimates of the TRGB level are based on small samples - is in good agreement with the calibration of Paper I, whose zero point relies on the huge sample of $\omega$ Cen stars by Pancino et al. (2000). This may be ascribed to the different distance scales adopted in the two cases. For example, the distance modulus of 47 Tuc adopted by DA90 is $(m-M)_{0}=13.39,0.08$ mag larger than the one assumed here (see below) and 0.11 mag larger than the one reported by F99. This difference in the distance scale may partially compensate the effect of the observational undersampling, reconciling the two calibrations, notwithstanding the difference in the observed TRGB magnitude.

\subsection{Distance modulus}

Though 47 Tuc is a nearby and very well studied cluster, there is significant scatter in the distance modulus estimates obtained by various authors with different methods (see Percival et al. 2002; Zoccali et al. 2001; Gratton et al. 2003, for discussions). The situation regarding the most recent determinations is clearly illustrated in Fig. 6, where the open symbols mark estimates based on (various) RR Lyrae/HB distance scales while filled symbols represent estimates that are independent of these scales. It is clear that there is little reason to prefer any given RR Lyrae/HB-independent distance estimate to the others. As a compromise solution, we adopt the average of the HB-independent estimates as our final $(m-M)_{0}$ value and the standard deviation as its uncertainty, reported as continuous and dashed lines in Fig. 6: $(m-M)_{0}=13.31 \pm 0.14$. We just note here that the adopted distance modulus is in good agreement with the estimates by F99 and F00 whose distance scale is fully compatible with our TRGB scale, as shown in Paper I. For consistency with F99 we also adopt $E(B-V)=0.04$, also in agreement with the most recent determination by Gratton et al. (2003) from the $B-V$ index (see their Table 3 ).

\section{Calibrations}

Following F99 and F00 we provide the calibration of the TRGB level in the $I, J, H$, and $K$ bands as a function of the global metallicity $[\mathrm{M} / \mathrm{H}]$. This parameter includes the contribution of the $\alpha$-elements according to the formula provided by Salaris et al. (1993). The observed properties of a given stellar population (at fixed age and helium content) do not depend simply on the iron abundance but also on the abundance of the $\alpha$-elements (see Salaris et al. 1993; Ferraro et al. 1999; Trager 2003 , for discussion and references), and some variations of the $[\alpha / \mathrm{Fe}]$ abundance ratio are observed among globulars (see, for example Carney 1996; Brown et al. 1997) and galaxies (see Shetrone et al. 2003; Bonifacio \& Caffau 2003; Trager 2003 , and references therein). For these reasons, $[\mathrm{M} / \mathrm{H}]$ provides a more suitable indicator of the global metal content to correlate with the observed properties of stars and stellar populations. We assume $[\alpha / \mathrm{Fe}]=+0.35$ for $\omega$ Cen, according to the recent review by Pancino (2003), and $[\alpha / \mathrm{Fe}]=+0.2$ for 47 Tuc, following Carney (1996) and the more recent results by Carretta et al. (2004). The final values of the global metallicity (computed with Eq. (1) in F99) are $[\mathrm{M} / \mathrm{H}]=-1.45 \pm 0.1$ and $[\mathrm{M} / \mathrm{H}]=-0.58 \pm 0.1$, for $\omega$ Cen and 47 Tuc, respectively.

The final calibrations are reported in Fig. 7. All the plots are on the same scale to better appreciate the different dependences on metallicity and the different degrees of uncertainty of the calibrations in the various passbands. The two observational points derived here are compared with the predictions of the theoretical models by Salaris \& Cassisi (1998, kindly provided in electronic form by Santino Cassisi). Panel (a) of 
47 Tucanae
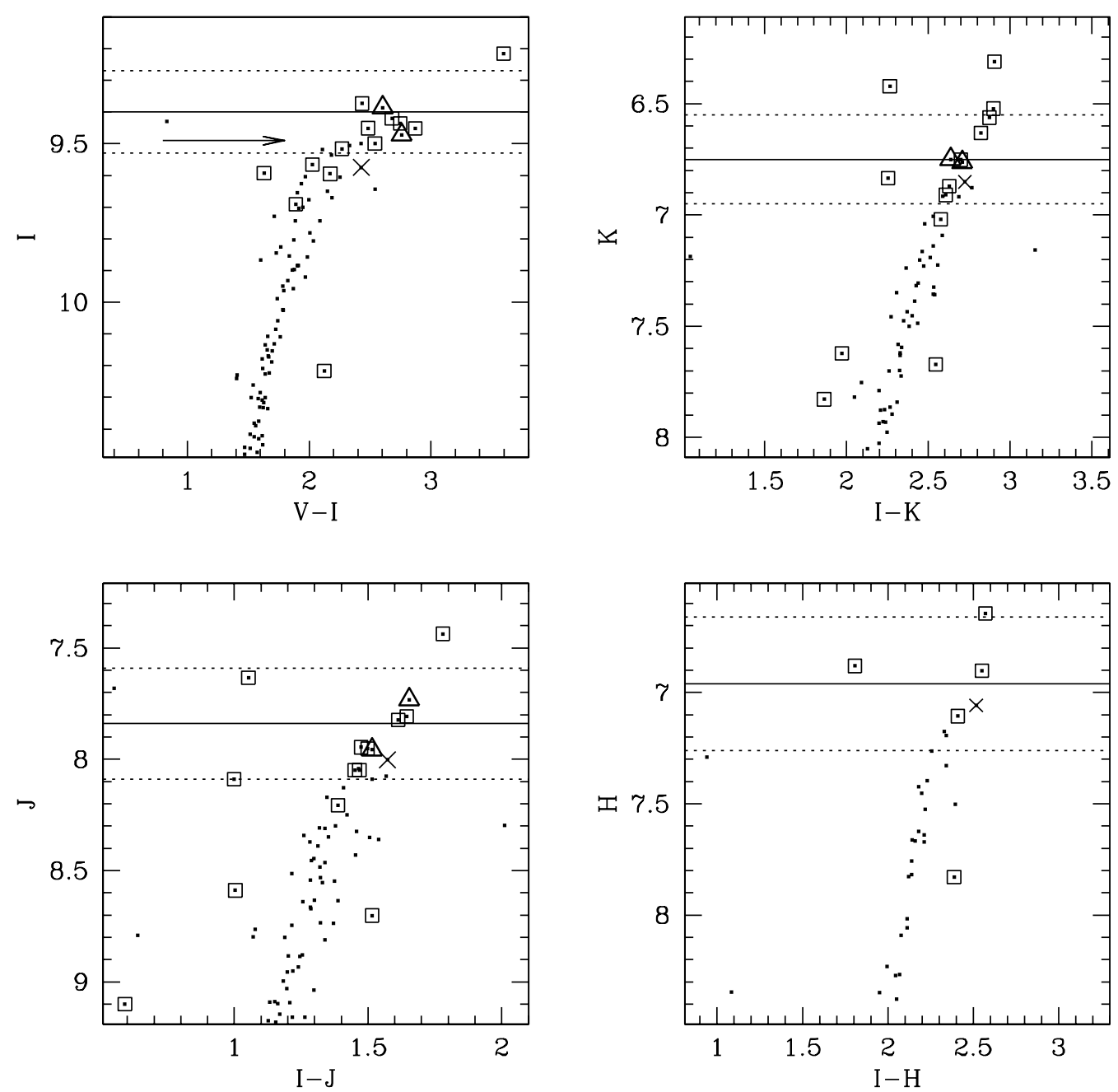

Fig. 5. The upper RGB of 47 Tucanae is shown in CMDs obtained from various combinations of the $I, K, J$, and $H$ photometry. The arrow in the $(I, V-I)$ CMD marks the position at which the TRGB is detected with the Sobel filter (Fig. 4) while the continuous line marks the true position of the TRGB once the correction for the poor sample is applied. The dotted lines enclose the $\pm 1 \sigma$ uncertainty range around this level. Open squares are long period variables. The open triangles are the brightest non-variable stars that have valid photometry in $I, J$ and $K$ and the cross is the brightest non-variable star that has $I$ and $H$ photometry.

Fig. 7 shows the calibration in the $I$ band. The quoted relation is the same as in Paper I, with a zero-point shift of +0.03 mag that we applied to achieve the best match to the observed points (thick continuous line). From the inspection of panel (b) it can be appreciated that the empirical relation for $M_{K}^{\mathrm{TRGB}}$ obtained by F00 provides a good fit to the observed points ${ }^{4}$. Hence we adopt it as our final calibration in the $K$ band.

${ }^{4}$ As discussed in Paper I, the RGB samples adopted by F00 to detect the TRGB in the $K$ passband are much richer than those used by DA90, for instance (see Sect. 4.2). Hence their estimates of the TRGB level are quite reliable, in general, and it is not surprising that their empirical relation is in good agreement with the accurate zero points derived here. However, it has to be noted that the F00 relation is 0.05 mag fainter, in $K$, with respect to our $\omega$ Cen zero point and the overall uncertainties in the single TRGB detections are significantly larger than what we obtained here for $\omega$ Cen and 47 Tuc. Finally, as also discussed in Paper I, in the attempt to provide calibrating relations
There are no available empirical calibrations of the TRGB in the $J$ and $H$ bands. However, since the considered models are in excellent agreement with our pillar references, we adopt a linear fit to the theoretical points as our final calibrations in these passbands (Fig. 7, panels (c) and (d)). This choice guarantees a full consistency with the observational constraints as well as a sound basis for the extrapolation of the calibration outside the metallicity range enclosed by the two data points.

The disagreement between our empirical calibration and the theoretical models by Salaris \& Cassisi (1998) in the $I$ band, already described and discussed in Paper I, is quite evident also

for extragalactic applications of the TRGB technique it is relevant, in our view, that the TRGB detection for the pillars of the calibration is based on the same operational definition of the observable that is used when dealing with distant galaxies, e.g. the cut-off of the RGB LF. This operational definition can be applied only to large RGB samples as those described in Sect. 2.2. 


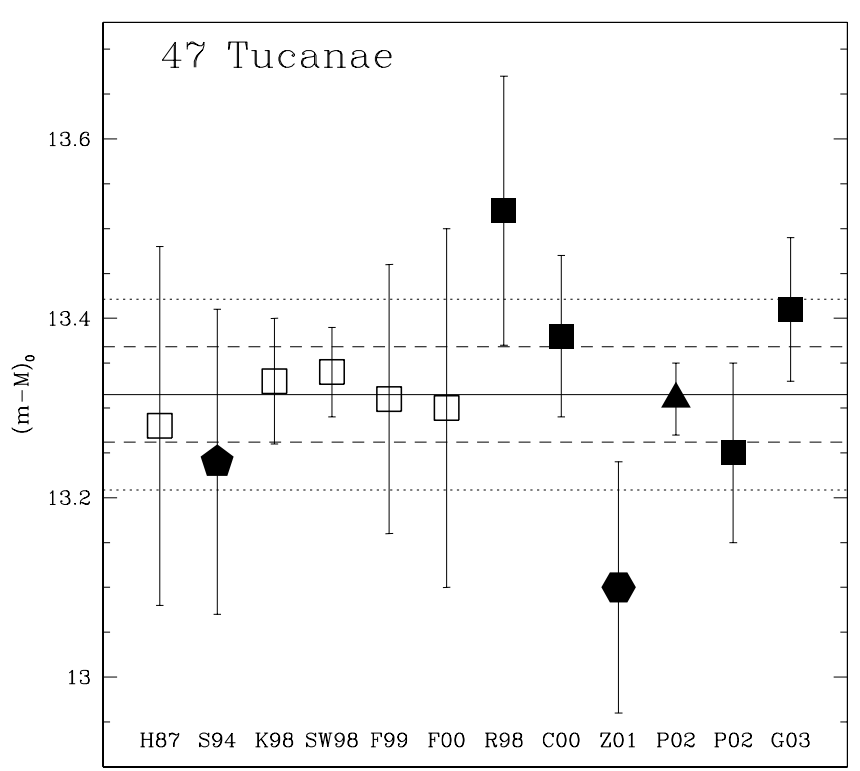

Fig. 6. Summary of recent estimates of the true distance modulus of 47 Tucanae. The open symbols refer to estimates relying on the RR Lyrae/HB distance scale while the filled symbols are from independent methods. In particular: filled squares = main sequence fitting; filled triangles = red clump (Hipparcos-calibrated); filled pentagons = Baade-Wesselink; filled hexagons $=$ white dwarf sequence fitting. The continuous line is the mean of the estimates that are independent of the RR Lyrae distance scale, the dashed and dotted lines enclose the $\pm 1 \sigma$ and $\pm 2 \sigma$ ranges about the mean, respectively.

in Fig. 7a. It is interesting to note that, nevertheless, model predictions are in excellent agreement with the observed points in all three NIR passband considered. This demonstrates that the difference found in the $I$ band is not due to a problem in our distance scale, since the reddening and distance assumptions are the same for all passbands, nor to an underestimate of the observed luminosity of the TRGB in $I$ due to under-sampling, since there are many more RGB stars in the available optical sample than in the NIR sample. At the present stage, we can only suggest that the most likely origin of the disagreement may reside in unsatisfactory color transformation of theoretical models in the $I$ band. Note, however, that there are also intrinsic $\left(M_{\text {bol }}\right)$ differences in the TRGB level prediction between the various theoretical models (see Salaris 2002, for references and discussion).

\subsection{The TRGB distance scale: The case of $L M C$}

The distance to the Large Magellanic Cloud (LMC) is the fundamental keystone of the extragalactic distance ladder and it has been the subject of considerable debate in the last decades. However, in recent years most of the results appear to converge toward a "standard value" $(m-M)_{0} \simeq 18.50$ (Alves 2004; Clementini et al. 2003). Hence, it is interesting to check if our independent TRGB distance scale provides a distance modulus of the LMC that is consistent with the standard value or not.

To do that, we take the observed apparent TRGB level in $I$, $J$ and $K$ provided by the DENIS survey (Cioni et al. 2000, hereafter $\mathrm{Ca}$ (00) and we adopt their value of $[\mathrm{M} / \mathrm{H}]=-0.7$ for the LMC. Given these assumptions we obtain an estimate of the true distance modulus and reddening from each couple of calibrating relations in the available passbands, i.e. $I$ and $J, I$ and $K, J$ and $K$ by finding the value of the reddening for which (exactly) the same $(m-M)_{0}$ is obtained from the two considered relations. The average and standard deviations of the three estimates obtained in this way are $\left\langle(m-M)_{0}\right\rangle=18.46 \pm 0.06$ and $\langle E(B-V)\rangle=0.07 \pm 0.06$. As expected, given the considerable uncertainties affecting the NIR relations presented in Fig. 7, the constraints on the reddening are quite loose but the (self-consistent) distance modulus obtained is in line with the standard value.

It is interesting to note that if we assume $E(B-V)=0.15$, as done by Cal00, the distance modulus we obtain from $I_{\mathrm{TRGB}}$ is considerably smaller than that obtained in the other passbands (18.28 compared to 18.43 and 18.42 , in $I, J$, and $K$ respectively). A full self consistency among the various passbands is achieved only if a smaller value of the reddening is adopted. However there are serious arguments favoring the adoption of a lower extinction (see Zaritsky 1999; Clementini et al. 2003; Dutra et al. 2001). In particular, Zaritsky (1999) has shown how the reddening issue may be critical in the estimate of the LMC distance modulus. According to this author, the amount of extinction varies significantly not only with the location within the galaxy but also as a function of the temperature of the considered stars. In particular, he demonstrated that the cool stars (as RGB stars) are less extincted than hot stars (essentially young Main Sequence stars, see Zaritsky 1999, for further details). According to Harris et al. (1997) and Zaritsky (1999) the reddening adopted by Cal00 is probably more appropriate for OB stars than for the cool RGB we are considering.

Sakai et al. (2000) provide a robust estimate of the dereddened apparent TRGB magnitude using a very large sample of LMC RGB stars. The observed I magnitude of each single star is corrected for reddening using the accurate extinction map provided by Harris et al. (1997) and Zaritsky (1999), and taking into account the temperature dependence of the extinction according to the prescriptions of Zaritsky (1999). Adopting their result $I_{\mathrm{TRGB}}^{0}=14.54 \pm 0.04$ we obtain $(m-M)_{0}=18.54 \pm 0.04$ (statistic) \pm 0.12 (systematic).

We conclude that our TRGB distance scale, whose zero point is based on the distance to $\omega$ Cen derived from the detached eclipsing binary OGLE-17, provides a distance modulus in full agreement with the standard value $(m-M)_{0} \simeq 18.50$. The same conclusion was reached by Alves (2004) using an independent dataset and the original $I$ calibration we provided in Paper I, which is essentially the same as shown in Fig. 7a, here. The same result is obtained independently of the adopted passband, once the correct reddening value has been adopted, showing that the different calibrations shown in Fig. 7 are fully self-consistent.

\section{Summary and conclusions}

We have refined the zero point of the calibration of the TRGB as a standard candle provided by Bellazzini et al. (2001, Paper I) adopting a more accurate estimate of the reddening 


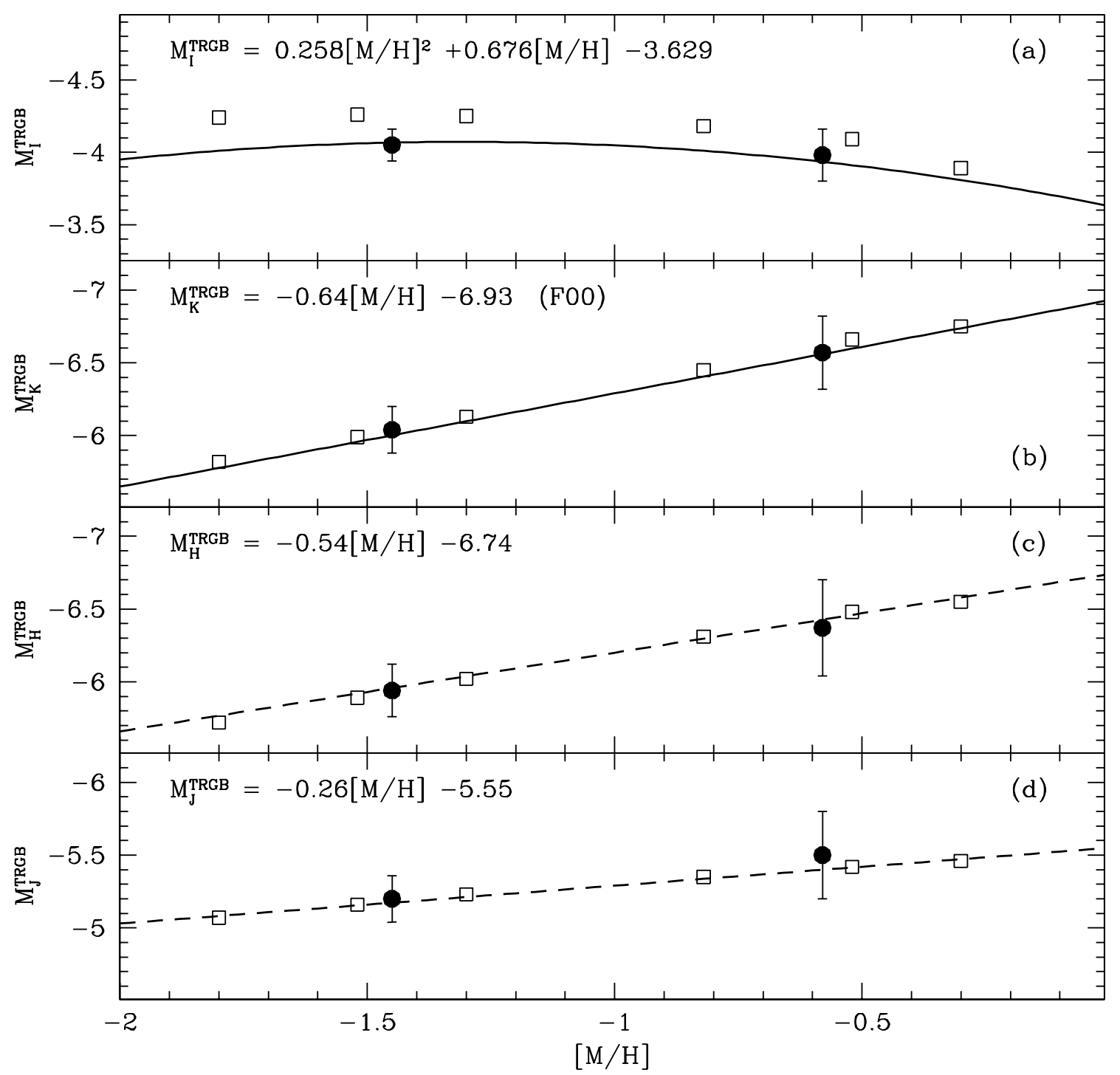

Fig. 7. Calibration of the absolute $I, K, H, J$ magnitude of the TRGB as a function of the global metallicity. The filled circles are the observational points, the open squares are the theoretical models by Salaris \& Cassisi (1998). Panel a): the continuous line is the empirical calibration derived in Paper I shifted by +0.03 mag (see Sect. 5). Panel b): the continuous line is the empirical calibration by F00. Panels c), d): the dashed lines are the linear fits to the model points.

to $\omega$ Centauri, the fundamental pillar of our distance scale. The calibration of Paper I has been accordingly revised and extended to NIR $(J, H, K)$ passbands using a new large photometric dataset of $\omega$ Cen giants taken from S03 and 2MASS.

A large optical and NIR photometric database has been assembled for the metal rich globular cluster 47 Tucanae. From this database we obtained the best estimate of the TRGB presently available for this cluster in $I, J, H$, and $K$, providing a second observational point for our TRGB calibrations, at $[M / H] \simeq-0.6$.

With this new observational material we provide new robust calibrations of the TRGB magnitude in the $I, J, H$, and $K$ bands as a function of the global metallicity. The obtained NIR calibrations are in excellent agreement with the theoretical predictions by Salaris \& Cassisi (1998), while the marginal discrepancy in the $I$ band already noted in Paper I persists and is probably due to the color transformation applied to theoretical models. It has also been shown that our calibrations self-consistently provide a distance modulus of the Large Magellanic Cloud in good agreement with the "standard value" (see Alves 2004; Walker 2003; Clementini et al. 2003).

With the present contribution, we think we have provided the "state of the art" instrument of stellar astrophysics needed to afford the study of extragalactic distances with the Population II distance scale (Walker 2003; Alves 2004), e.g. a fundamental complement and consistency test for Cepheid and SNIa distance scales.

Acknowledgements. We acknowledge the financial support to this research by the Italian Ministero dell'Universitá e della Ricerca Scientifica (MURST). We are grateful to S. Cassisi for providing models predictions in electronic form and for many insightful discussion. Part of the data analysis has been performed with software 
developed by P. Montegriffo at the Osservatorio Astronomico di Bologna (INAF). This research has made use of ESO Archive data taken with the WFI at the MPI/ESO $2.2 \mathrm{~m}$ Telescope at the La Silla Observatory. This publication makes use of data products from the Two Micron All Sky Survey, which is a joint project of the University of Massachusetts and the Infrared Processing and Analysis Center/California Institute of Technology, funded by the National Aeronautics and Space Administration and the National Science Foundation. This research has made use of NASA's Astrophysics Data System Abstract Service.

\section{References}

Alves, D. 2003, in Stellar candles for the extragalactic distance scale, ed. D. Alloin, \& W. Gieren, Springer, Lect. Notes Phys., in press [arXiv:astro-ph/0303035]

Alves, D. 2004, in Extragalactic Binaries, IAU XXV General Assembly, ed. A. Gimenez, \& I. Ribas, New Astron. Rev., 48, 659

Buzzoni, A. 1989, ApJS, 71, 817

Buzzoni, A. 2002, AJ, 123, 1188

Barker, M. K., Sarajedini, A., \& Harris, J. 2004, ApJ, 606, 869

Bellazzini, M., Ferraro, F. R., \& Pancino, E. 2001, ApJ, 556, 635

Bellazzini, M., Ferraro, F. R., Origlia, L., et al. 2002, AJ, 124, 3222

Bonifacio, P., \& Caffau, E. 2003, A\&A, 399, 1183

Brown, J. A., Wallerstein, G., \& Zucker, D. 1997, AJ, 114, 180

Cacciari, C. 1999, in Harmonizing cosmic distance scales in a post-HIPPARCOS Era, ed. D. Egret, \& A. Heck (S. Francisco: ASP), ASP Conf. Ser., 167, 140

Caputo, F., Degl'Innocenti, S., \& Marconi, M. 2002, in $\omega$ Centauri: A unique window into astrophysics, ed. F. van Leeuwen, J. Hughes, \& G. Piotto (S. Francisco: ASP), ASP Conf. Ser., 265, 185

Carney, B. W. 1996, PASP, 108, 900

Carpenter, J. M. 2001, AJ, 121, 2851

Carretta, E., \& Gratton, R. 1997, A\&AS, 121, 95

Carretta, E., Gratton, R. G., Clementini, G., \& Fusi Pecci, F. 2000, ApJ, 533, 215 (C00)

Carretta, E., Gratton, R. G., Bragaglia, A., Bonifacio, P., \& Pasquini, L. 2004, A\&A, 416, 925

Cioni, M.-R. L., van der Marel, R. P., Loup, C., \& Habing, H. J. 2000, A\&A, 359, 601

Clement, C. 1997, AAS Newsl., 84, 15

Clementini, G., Gratton, R., Bragaglia, A., et al. 2003, AJ, 125, 1309

Cutri, et al. 2003, Explanatory Supplement to the 2MASS All Sky Data Release http://www. ipac.caltech.edu/2mass/ releases/allsky/doc/explsup.html

Da Costa, G. S., \& Armandroff, T. A. 1990, AJ, 100, 162 (DA90)

Dean, J. F., Warren, P. R., \& Cousins, A. W. J. 1978, MNRAS, 183, 569

Dutra, C. M., Bica, E., Clariá, J. J., Piatti, A. E., \& Ahumada, A. V. 2001, A\&A, 371, 895

Elias, J. H., Frogel, J. A., Hyland, A. R., \& Jones, T. J. 1983, AJ, 88, 1027

Ferraro, F. R., Messineo, M., Fusi Pecci, F., et al. 1999, AJ, 118, 1738 (F99)

Ferraro, F. R., Montegriffo, P., Origlia, L., \& Fusi Pecci, F., et al. 2000, AJ, 119, 1282 (F00)

Ferraro, F. R., Pancino, E., \& Bellazzini, M. 2002, in $\omega$ Centauri: A Unique Window into Astrophysics, ed. F. van Leeuwen, J. Hughes, \& G. Piotto (S. Francisco ASP), ASP Conf. Ser., 265, 407

Gratton, R. G., Bragaglia, A., Carretta, E., et al. 2003, A\&A, 408, 529 (G03)
Harris, W. E. 1996, AJ, 112, 1487

Harris, J., Zaritsky, D., \& Thompson, I. 1997, AJ, 114, 1002

Hesser, J. E., Harris, W. E., VandenBerg, D. A., et al. 1987, PASP, 99, 739 (H87)

Hilker, M., \& Richtler, T. 2000, A\&A, 362, 895

Hughes, J., \& Wallerstein, G. 2000, AJ, 119, 1225

Kaluzny, J., Wysocka, A., Stanek, K. Z., \& Krzeminski, W. 1998, Acta Astron., 48, 439 (K98)

Kovàcs, G. 2002, in $\omega$ Centauri: A unique window into astrophysics, ed. F. van Leeuwen, J. Hughes, \& G. Piotto (S. Francisco: ASP), ASP Conf. Ser., 265

Lee, Y. W., Demarque, P., \& Zinn, R. 1990, ApJ, 350, 155

Lee, M. G., Freedman, W. L., \& Madore, B. F. 1993, ApJ, 417, 553 (LFM93)

Lub, J. 2002, in $\omega$ Centauri: A unique window into astrophysics, ed. F. van Leeuwen, J. Hughes, \& G. Piotto (S. Francisco: ASP), ASP Conf. Ser., 265, 95

Madore, B. F., \& Freedman, W. L. 1995, AJ, 109, 1645 (MF95)

Madore, B. F., \& Freedman, W. L. 1998, in Stellar astrophysics for the Local Group, ed. A. Aparicio, A. Herrero, \& F. Sanchez (Cambridge: Cambridge University Press), 305

Maíz-Apellániz, J., Cieza, L., \& MacKenty, J. W. 2002, AJ, 123, 1601

Mendez, B., Davis, M., Moustakas, J., et al. 2002, AJ, 124, 213

Montegriffo, P., Ferraro, F. R., Fusi Pecci, F., \& Origlia, L. 1995, MNRAS, 276, 739

Norris, J. E., \& Da Costa, G. S. 1995, ApJ, 447, 680

Norris, J. E., Freeman, K. C., \& Mighell, K. L. 1996, ApJ, 462, 241

Olech, A., Kaluzny, J., Thompson, I. B., \& Schwarzenberg-Czerny, A., MNRAS, 345, 86

Origlia, L., Ferraro, F. R., Bellazzini, M., \& Pancino, E. 2003, ApJ, 591, 916

Pancino, E., Ferraro, F. R., Bellazzini, M., Piotto, G., \& Zoccali, M. 2000, ApJ, 534, L83

Pancino, E., Pasquini, L., Hill, V., Ferraro, F. R., \& Bellazzini, M. 2002, ApJ, 586, L101

Pancino, E. 2003, Ph.D. Thesis, Bologna University

Percival, S. M., Salaris, M., van Wyk, F., \& Kilkenny, D. 2002, ApJ, 573, 174 (P02)

Reid, I. N. 1998, AJ, 115, 204 (R98)

Renzini, A. 1998, AJ, 115, 2459

Renzini, A., \& Buzzoni, A. 1986, in Spectral evolution of galaxies, ed. C. Chiosi, \& A. Renzini (Dordercht: Reidel), 135

Renzini, A., \& Fusi Pecci, F. 1988, ARA\&A, 26, 199

Rey, S.-C., Lee, Y. W., Joo, J.-M., Walker, A., \& Baird, S. 2000, AJ, 119,1824

Rieke, G. H., \& Lebofsky, M. J. 1985, ApJ, 288, 618

Robin, A. C., Reylé, C., Derrière, S., \& Picaud, S. 2003, A\&A, 409, 523

Rood, R. T., \& Crocker, D. A. 1997, http://www.astro. virginia.edu/rtr/papers/mpsvi97.ps.gz

Sakai, S., Madore, B. F., \& Freedman, W. L. 1996, ApJ, 461, 713 (SMF96)

Sakai, S., Zaritsky, D., \& Kennicut, R. C. Jr. 2000, AJ, 119, 1197

Salaris, M. 2002, in Observed HR diagrams and stellar evolution: the interplay between observational constraints and theory, ed. T. Lejeune, \& J. Fernandes (San Francisco: ASP), ASP Conf. Ser., 274,50

Salaris, M., \& Cassisi, S. 1998, MNRAS, 298, 166 (SC98)

Salaris, M., \& Weiss, A. 1998, A\&A, 335, 943 (SW98)

Salaris, M., Chieffi, A., \& Straniero, O. 1993, ApJ, 414, 580

Salaris, M., Cassisi, S., \& Weiaa, A. 2002, PASP, 114, 375 
Sarajedini, A., \& Layden, A. C. 1995, AJ, 109, 1086

Schechter, P., Mateo, M., \& Saha, A. 1993, PASP, 105, 1342

Shetrone, M., Venn, K. A., Tolstoy, E., Primas, F., Hill, V., \& Kaufer, A. 2003, AJ, 125, 684

Smith, V. V., Hinkle, K. H., Cunha, K., et al. 2002, AJ, 124, 3241

Sollima, A., Ferraro, F. R., Origlia, L., Pancino, E., \& Bellazzini, M. 2003, A\&A, submitted

Stetson, P. 2000, PASP, 112, 925

Storm Nordström, B., Carney, B. W., \& Andersen, J. 1994, A\&A, 291, 121 (S94)

Suntzeff, N. B., \& Kraft, R. P. 1996, AJ, 111, 1913

Thompson, I. B., Kaluzny, J., Pych, W., et al. 2001, AJ, submitted [arXiv: astro-ph/0012493]

Tosi, M., Sabbi, E., Bellazzini, M., et al. 2001, AJ, 122, 1271
Trager, S. 2003, in Origin and evolution of the elements, ed. A. McWilliam, \& M. Rauch (Cambridge: Cambridge University Press), Carnegie Obs. Astroph. Ser., 4, in press [arXiv: astro-ph/0307069]

Valenti, E., Ferraro, F. R., Perina, S., \& Origlia, L. 2004, A\&A, 419, 139

van Leeuwen, F., Poole, R. S., Reijns, R. A., Freeman, K. C., \& de Zeeuw, P. T. 2000, A\&A, 360, 472

Walker, A. R. 2003, in Stellar candles for the extragalactic distance scale, ed. D. Alloin, \& W. Gieren (Springer), Lect. Notes Phys., 635,265

Zaritsky, D. 1999, AJ, 118, 2824

Zinn, R., \& West, M. J. 1984, ApJS, 55, 45

Zoccali, M., Renzini, A., Ortolani, S., et al. 2001, ApJ, 553, 733 (Z01) 